\title{
Ultrastructure of a Somatic Spine Mat for Nicotinic Signaling in Neurons
}

\author{
Richard D. Shoop, ${ }^{1}$ Eduardo Esquenazi, ${ }^{2,3}$ Naoko Yamada, ${ }^{2,3}$ Mark H. Ellisman,, ${ }^{2,3}$ and Darwin K. Berg ${ }^{1}$ \\ ${ }^{1}$ Neurobiology Section, Division of Biology, ${ }^{2}$ Department of Neuroscience, and the ${ }^{3}$ National Center for Microscopy and \\ Imaging Research, University of California, San Diego, La Jolla, California 92093-0357
}

Chick ciliary neurons have somatic spines grouped in discrete clumps or mats tightly folded against the soma and enriched in nicotinic receptors containing $\alpha 7$ subunits. An embryonic ciliary neuron has one to two dozen such spine mats, all overlaid by a large presynaptic calyx engulfing the cell. Three-dimensional tomographic reconstruction from serial thick sections revealed 13 somatic spines in one complete spine mat on a ciliary neuron late in embryogenesis. The spines varied in morphology and usually were branched but had numerous similarities to dendritic spines, including mean length, volume, surface area, presence of endoplasmic reticulum, and occasional multivesicular bodies. The spines invariably were connected to the soma via a narrow neck of $\sim 0.2 \mu \mathrm{m}$ in diameter as found for dendritic spines, suggesting restricted access from spine lumen to soma. A prominent difference between dendritic and somatic spines is the absence of postsynaptic densities from most somatic spines both on embryonic and adult ciliary neurons. Transmitter access to receptors on the spines may occur either by lateral diffusion from release sites over nearby postsynaptic densities or by release directly onto spines from the overlying calyx lined with vesicles. The latter is less likely in the adult, where some spines are adjacent to but not overlaid by vesicle-enriched presynaptic structures. The anatomical configuration of spine mats suggests coordinate spine activation by transmitter release into a confined volume while spine morphology is used to control the chemical consequences of synaptic signaling.

Key words: nicotinic; spine; receptor; ciliary; ganglion; synapse
Dendritic spines have been the subject of intense investigation in recent years. Initially described by Ramon y Cajal (1893), the spines are small protrusions distributed along the dendritic shaft (for review, see Harris, 1999; Matus, 2000). In the CNS they commonly receive a single excitatory glutamatergic synapse and have a prominent postsynaptic density (PSD). The shape of the spine is thought to promote a dual synaptic function, namely ready transmission of electrical signals through the narrow spine neck into the parent dendrite but local retention within the spine of chemical signals that depend on diff usion for propagation. This is particularly important in the case of synaptically triggered postsynaptic calcium elevations that cause synaptic plasticity. Confining the calcium elevations to individual spines makes possible synapse-specific plasticity (for review, see Yuste et al., 2000; Sabatini et al., 2001).

Somatic spines have been seen both on CNS neurons, as in the case of dentate gyrus granule cells (Stirling and Bliss, 1978; Seress and Ribak, 1985; Bundman et al., 1994; Wenzel et al., 1994), and on autonomic neurons from a variety of ganglia (Piezzi and Rodriguez-Echandia, 1968; Watanabe, 1971; Smolen, 1988; Robertson and Jackson, 1996). On chick ciliary neurons the somatic spines are grouped in clusters and are interwoven and tightly folded down on the soma to form mats (Shoop et al., 1999). By

\footnotetext{
Received Sept. 10, 2001; revised Oct. 17, 2001; accepted Oct. 26, 2001.

Grant support was provided by National Institutes of Health Grants NS12601, NS35469, and RR04050 and by Tobacco-Related Disease Research Program Grant 9RT-0221. We thank Dr. Maryann E. Martone for helpful advice throughout and for assistance in preparing the final figures.

Correspondence should be addressed to Darwin K. Berg, Neurobiology Section, Division of Biology, 0357, University of California, San Diego, 9500 Gilman Drive, La Jolla, CA 92093-0357. E-mail: dberg@ucsd.edu.

Copyright (C) 2002 Society for Neuroscience $0270-6474 / 02 / 220748-09 \$ 15.00 / 0$
}

late embryogenesis the individual neurons can have one to two dozen such mats covered by the presynaptic calyx that encapsulates the soma and that is packed with synaptic vesicles. Occasional PSDs can be seen on the spines. Nicotinic transmission through the calyx synapse is mediated by nicotinic acetylcholine receptors containing the $\alpha 7$ gene product ( $\alpha 7$-nAChRs) concentrated on the spines but excluded from PSDs (Jacob and Berg, 1983; Loring et al., 1985; Wilson Horch and Sargent, 1995; Zhang et al., 1996; Ullian et al., 1997; Chang and Berg, 1999; Shoop et al., 1999) and by less abundant heteromeric receptors containing the $\alpha 3, \beta 4, \alpha 5$, and sometimes $\beta 2$ gene products ( $\alpha 3^{*}$-nAChRs) present both in PSDs and on the spines (Jacob et al., 1984; Loring and Zigmond, 1987; Vernallis et al., 1993; Conroy and Berg, 1995; Williams et al., 1998). In the adult the presynaptic calyx transforms into large boutons (De Stefano et al., 1993), but the high levels of ganglionic $\alpha 7$-nAChRs are retained (Chiappinelli and Giacobini, 1978).

The present study was undertaken to determine the ultrastructure of a complete spine mat with the use of electron microscopy (EM) and three-dimensional tomographic reconstructions of images from serial thick sections. The goals were to determine the shape, size, and number of spines in a mat, the proximity of spines to synaptic vesicles, and the frequency of PSDs on spines. In addition, comparisons were made between embryonic and adult ganglia to determine which features were developmentally stable. The results demonstrate a complex mat of spines with classical morphologies and collective stability but few PSDs, even in adulthood. Spine proximity to synaptic vesicles may decrease with age, suggesting that the activation of receptors on spines in mature ganglia would require lateral diff usion from distal release sites. 

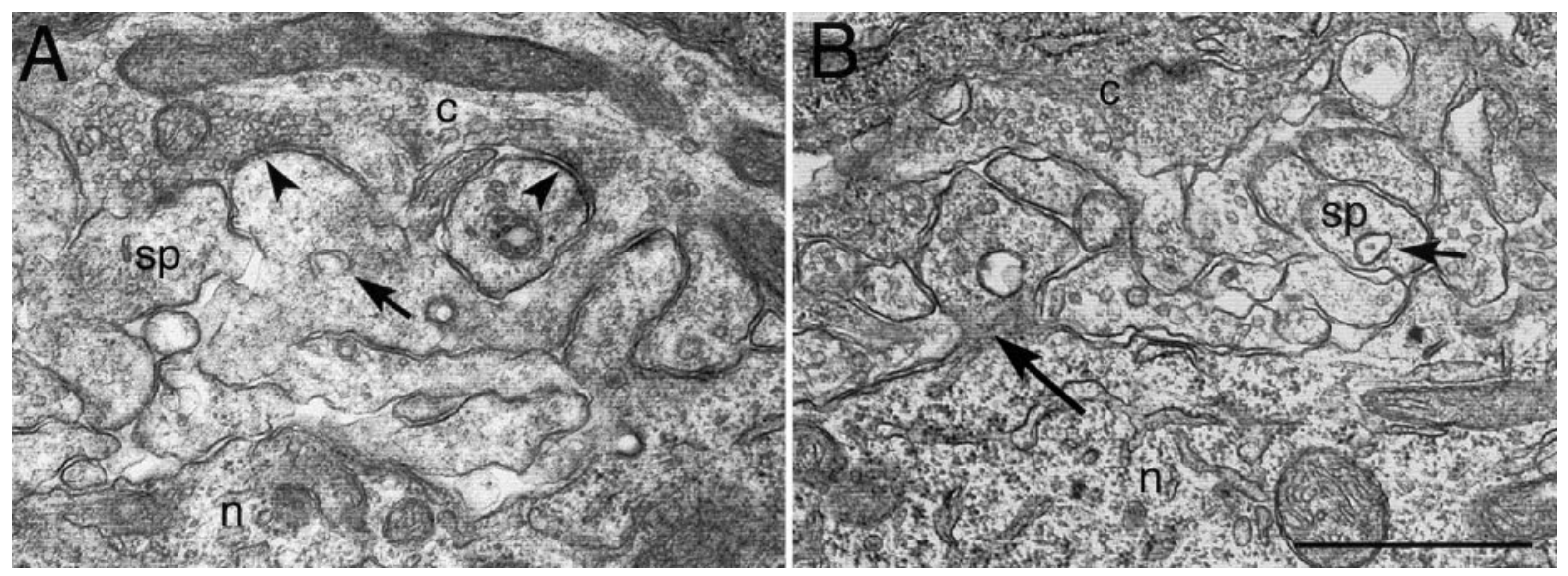

Figure 1. Traditional EM on thin sections of E15 ciliary neurons showing spines containing PSDs and ER and being surrounded by vesicle-filled presynaptic calyx. $A$, Spine mat showing spines $(s p)$ with PSDs (arrowheads) and ER (small arrow) lying between the neuron soma $(n)$ and presynaptic calyx $(c)$. A calyx protrusion extends into the mat but is distinct from the two spines, although the intervening membranes are not always readily visible in isolated thin sections. $B$, A spine mat lying between a neuron soma $(n)$ and overlying calyx $(c)$ and showing a spine neck connecting a spine to the soma (large arrow) plus a spine $(s p)$ with ER (small arrow). Scale bar, $500 \mathrm{~nm}$.

\section{MATERIALS AND METHODS}

Sample preparation. Embryonic day (E) 15 chicks or 2-year-old adult chickens were perfusion-fixed with $2 \%$ paraformaldehyde plus $2 \%$ glutaraldehyde in cacodylate buffer, $\mathrm{pH}$ 7.4. Ciliary ganglia were removed, transferred to fresh fixative, and incubated for $3 \mathrm{hr}$ at room temperature. Adult ciliary ganglia were cut into $1 \mathrm{~mm}^{3}$ pieces. After being rinsed several times in $0.1 \mathrm{M}$ sodium cacodylate buffer, $\mathrm{pH} 7.4$, the tissue was treated for $30 \mathrm{~min}$ with $2 \%$ osmium tetroxide in $0.1 \mathrm{M}$ sodium cacodylate and then counterstained with uranyl acetate. The ganglia were dehydrated in a series of ethanol solutions followed by two rinses in acetone, infiltrated with Durcupan ACM resin (Electron Microscopy Sciences, Fort Washington, PA), allowed to polymerize for $24 \mathrm{hr}$ at $60^{\circ} \mathrm{C}$, and then sectioned. For serial tomography a continuous series of $101-\mu \mathrm{m}$-thick sections was prepared to encompass a complete spine mat. For traditional thin section analysis 100 -nm-thick sections were made.

Electron microscopy and tomographic reconstruction. Thin-sectioned material was examined with a JEOL 100CX electron microscope. Serial $1-\mu$ m-thick sections were examined with a JEOL 4000EX microscope operating at $400 \mathrm{keV}$, as described previously (Soto et al., 1994; Shoop et al., 1999). A somatic spine mat was identified in one of the middle sections of the series. Images were taken at $10,000 \times$ magnification in the same region of all of the serial sections containing the spine mat. Volume content of the thick sections was revealed by tomographic reconstruction. Each $1-\mu \mathrm{m}$-thick section was rotated through $120^{\circ}$, and EM images were taken at $2^{\circ}$ increments. The 61 tilt images were digitized, aligned, and back-projected to generate the volume information by using software designed and written at the National Center for Microscopy and Imaging Research (La Jolla, CA) and described by Perkins et al. (1997).

Six $1 \mu \mathrm{m}$ serial sections encompassed the complete somatic spine mat and were combined into one volume for segmentation and analysis. Individual objects were traced (segmented) through all of the 720 sections contained in the volume with the Xvoxtrace software (developed by S. Lamont, National Center for Microscopy and Imaging Research). This information was used to construct three-dimensional graphic representations with the Synu software package (Hessler et al., 1992). Colors were chosen manually and were used consistently to represent each spine and neuronal element illustrated for a given spine mat.

Calculations. Somatic spine length, volume, and surface area were measured from the segmented volumes with XDend software, and spine neck diameters were measured with Analyze AVW software (National Center for Microscopy and Imaging Research). Length was calculated as the mean length of the longest continuous path from the spine neck to the tip of the spine. Significant deviations from this continuous path were counted as branches. Volume and surface were calculated directly on the basis of the traced morphologies after pixel size was converted to dimensions in micrometers.

Materials. White Leghorn chick embryos and adult chickens (2 years old) were obtained locally. Embryos were maintained at $37^{\circ} \mathrm{C}$ in a humidified incubator. All other reagents were purchased from Sigma (St. Louis, MO) unless otherwise indicated.

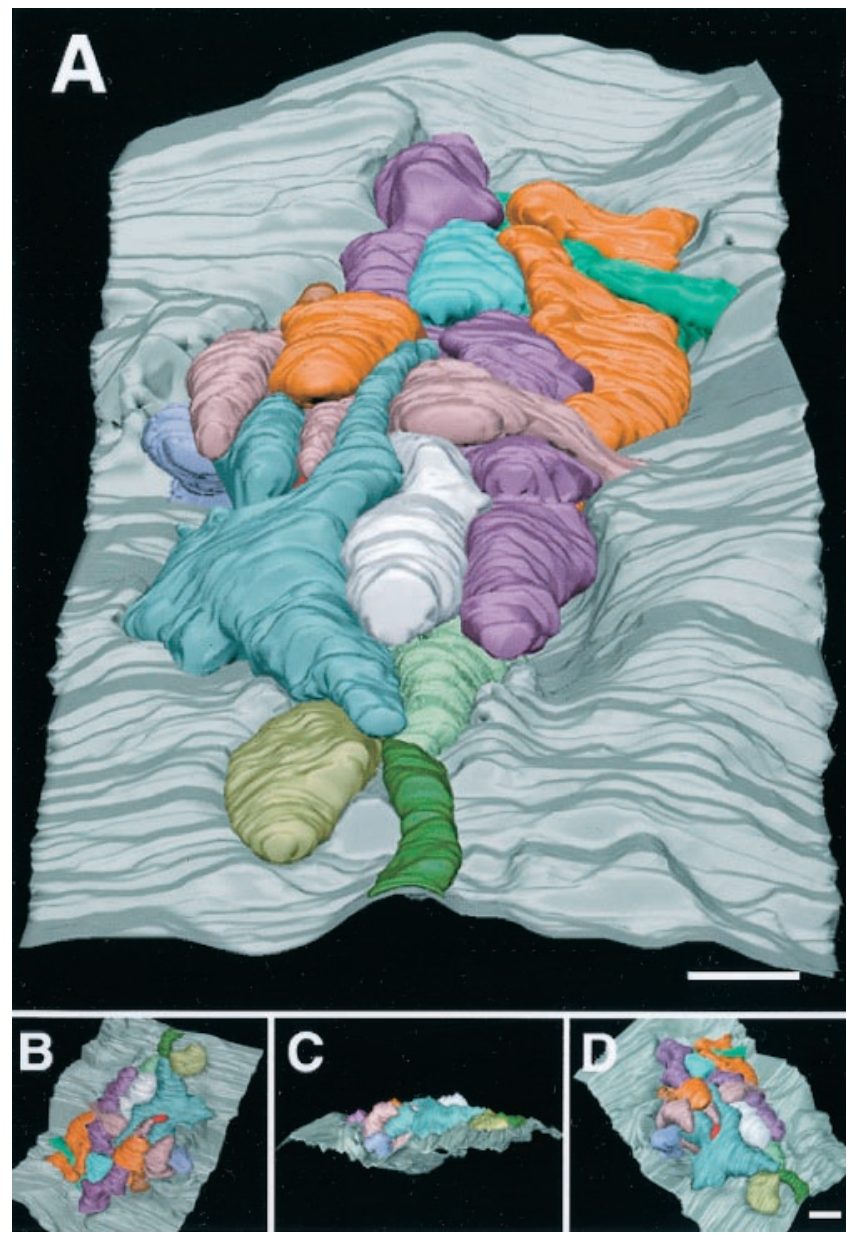

Figure 2. A complete spine mat with 13 somatic spines on an E15 ciliary neuron. $A$, The spine mat is shown at a $45^{\circ}$ angle from the horizontal, with spines individually color-coded (in this and subsequent figures) against the soma membrane shown in gray and covering a $24 \mu \mathrm{m}^{2}$ area. $B$, Rotating the spine mat by $200^{\circ}$ shows the opposite side of the spine mat. $C$, Tilting the neuronal surface to examine the membrane on edge shows that the somatic spines lie in a cavity in the neuronal soma and also project above the soma surface. $D$, Rotating the view in $C$ by $90^{\circ}$ in the horizontal gives a view from directly above the spine mat. Scale bars, $500 \mathrm{~nm}$. 


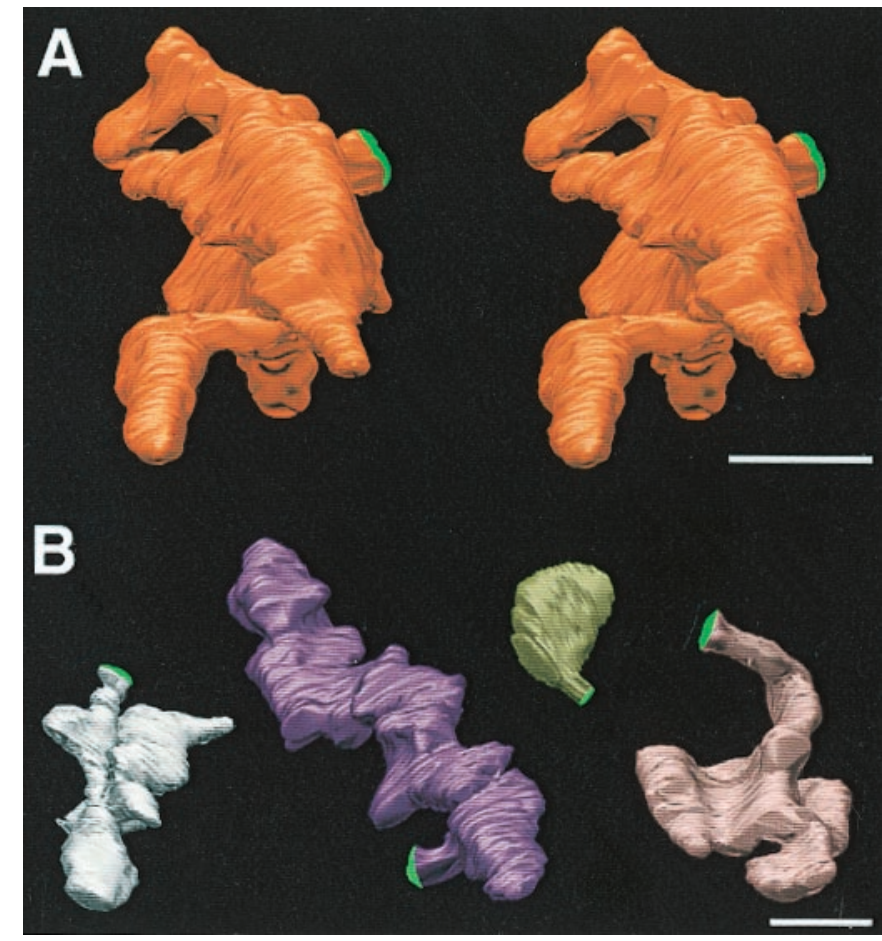

Figure 3. Selected individual spines illustrating the diverse morphologies. $A$, One of the largest and most branched somatic spines of the E15 mat, shown as a stereo pair. $B$, Four additional examples of somatic spines from the same mat, each shown at three-fourths the size of that in $A$. Spine necks and attachment sites are indicated in green. Scale bars, $500 \mathrm{~nm}$.

\section{RESULTS}

Traditional EM of thin sections suggests a complex structure for spine mats on ciliary neurons. Numerous somatic spine cross sections can be distinguished in an image at E15 (Fig. 1). The spines appear closely folded together and are overlaid completely with presynaptic structures packed with synaptic vesicles. PSDs can be seen occasionally on a spine, and some spines contain clearly visible internal membrane structures representing smooth endoplasmic reticulum (ER). The neck of the spine, when seen emerging from the soma, appears small. Similar images have been reported previously (Jacob and Berg, 1983; Shoop et al., 1999).

Three-dimensional structure of an E15 somatic spine mat

Three-dimensional tomographic reconstruction from serial section EM was used to determine the ultrastructure of a spine mat on an E15 ciliary neuron. Six $1-\mu$ m-thick serial sections were sufficient to encompass the complete spine mat. The volume comprised $96 \mu \mathrm{m}^{3}(6 \times 4 \times 4 \mu \mathrm{m})$ and was divided into 720 reconstructed individual slices. Objects of interest within each slice were traced, and the information was used to construct the object across slices. For clarity of representation the postsynaptic membrane was segmented into individual somatic spines and soma membrane. The analysis revealed 13 distinct spines comprising the complete mat (Fig. 2). Each spine is represented by a distinctive color, and the color code is maintained throughout Figures 2-6 and Table 1 .

The spines display a variety of shapes, including extended branches, and are intertwined intimately. Together the spines represent a closely packed mat tightly layered on the postsynaptic

\section{Table 1. Morphological characteristics of individual somatic spines in one complete spine mat on an E15 ciliary neuron.}

\begin{tabular}{|c|c|c|c|c|c|c|c|c|}
\hline & $\begin{array}{l}\text { Length } \\
(\mu \mathrm{m})\end{array}$ & $\begin{array}{l}\text { Volume } \\
\left(\mu \mathrm{m}^{3}\right)\end{array}$ & $\begin{array}{c}\text { Surface Area } \\
\left(\mu \mathrm{m}^{2}\right)\end{array}$ & $\begin{array}{c}\text { \% Surface } \\
\text { Exposed }\end{array}$ & $\begin{array}{l}\text { Num. } \\
\text { Branches }\end{array}$ & $\begin{array}{l}\text { Spine neck } \\
\text { Area }\left(\mu \mathrm{m}^{2}\right)\end{array}$ & PSD & ER \\
\hline Spine 0 & 0.64 & 0.01 & 0.23 & $51.5 \%$ & 1 & 0.034 & no & no \\
\hline Spine 1 & 0.88 & 0.04 & 0.49 & $38.0 \%$ & 1 & 0.030 & no & no \\
\hline - Spine 2 & 3.08 & 0.06 & 1.06 & $13.2 \%$ & 1 & 0.045 & no & no \\
\hline Spine 3 & 4.30 & 0.19 & 3.19 & $24.4 \%$ & 3 & 0.025 & yes & no \\
\hline$\square$ Spine 4 & 4.36 & 0.12 & 1.78 & $15.5 \%$ & 4 & 0.026 & yes & yes \\
\hline - Spine 5 & 5.77 & 0.26 & 3.50 & $32.4 \%$ & 3 & 0.033 & yes & yes \\
\hline Spine 6 & 0.88 & 0.04 & 0.48 & $23.4 \%$ & 1 & 0.045 & no & no \\
\hline Spine 7 & 5.49 & 0.21 & 3.01 & $18.3 \%$ & 4 & 0.035 & yes & no \\
\hline 풀 Spine 8 & 0.93 & 0.02 & 0.32 & $15.2 \%$ & 1 & 0.034 & no & yes \\
\hline Spine 9 & 2.78 & 0.06 & 1.33 & $8.01 \%$ & 2 & 0.032 & no & yes \\
\hline Spine 10 & 1.21 & 0.04 & 0.76 & $23.4 \%$ & 1 & 0.037 & no & yes \\
\hline Spine 11 & 5.25 & 0.28 & 3.46 & $24.7 \%$ & 6 & 0.027 & no & yes \\
\hline Spine 12 & 2.37 & 0.07 & 1.21 & $20.1 \%$ & 2 & 0.021 & no & yes \\
\hline Mean & 3.1 & 0.11 & 1.6 & $24 \%$ & 2.3 & 0.033 & $31 \%$ & $54 \%$ \\
\hline \pm SEM & \pm 2.2 & \pm 0.09 & \pm 1.3 & $\pm 12 \%$ & & \pm 0.007 & & \\
\hline
\end{tabular}

Measurements were made on each of the 13 reconstructed somatic spines present in the E15 mat. The color code identifies the spines illustrated in figures 2-6. The percent surface exposed is the percentage of the total spine surface in contact with presynaptic calyx containing synaptic vesicles. Spine neck area is the size of the opening of the spine lumen to the neuronal soma measured at its narrowest region. 



Figure 4. Characteristic attachment sites and contents of somatic spines on an E15 ciliary neuron. $A$, Soma membrane (gray; as in Fig. $2 A$ ) with the spines removed and the attachment sites indicated in green. The attachment sites are approximately the same size and are distributed nonuniformly over the entire surface area supporting the spine mat. $B, C$, Examples from reconstructed cross sections showing spine necks and attachment sites (arrowheads) to the soma. Multivesicular bodies (asterisk) were seen sometimes. D, E, Examples from reconstructed cross sections showing ER (arrowheads), which was extensive in seven of the 13 spines. F, Semitransparent rendition of spine 11 to illustrate the internal distribution of ER (gray) Scale bars: $A, 500 \mathrm{~nm} ; B-F, 200 \mathrm{~nm}$.

surface. The mat resides in a shallow depression in the postsynaptic cell but also protrudes above the surface. Rotating the image through different angles provides different perspectives of the mat and reveals the architecture (Fig. 2).

\section{Spine morphologies and connections with the soma}

A variety of shapes was seen for spines within the same mat. For illustrative purposes several are shown in isolation (Fig. 3). One of the more complex spines had six separate branches and is presented as a stereo pair (Fig. 3A). The spine neck (in green) connecting the spine to the soma membrane can be found at the top right. Other spines also were branched often, and usually the branches were interdigitated; occasional short stubby spines could be seen (Fig. $3 B$ ). The average spine length was $\sim 3 \mu \mathrm{m}$, and the average volume was $\sim 0.11 \mu \mathrm{m}^{3}$. Most had no clear spine head, in contrast to dendritic spines (Harris and Kater, 1994). Some of the structural features described here and below have been measured for the individual spines and tabulated for comparison (Table 1).

Despite the large variation in spine morphology the spines showed remarkable consistency in the size and shape of their connections to the postsynaptic neuron. Removing the spines from the composite image and marking their points of origin (Fig. 4, in green) shows that the 13 connections to the soma were distributed throughout the indented area supporting the spine mat (Fig. 4A). The spines were constricted at the point of fusion with the soma (Fig. 4B,C). The cross-sectional area connecting the spine lumen to the soma interior averaged $\sim 0.03 \mu \mathrm{m}^{2}$ (Table 1). A mean neck diameter was calculated from direct independent measurements as being $0.18 \pm 0.01 \mu \mathrm{m}$ (mean $\pm \mathrm{SEM} ; n=13)$. Occasionally, a multivesicular body was apparent within the spine (Fig. 4C). Multivesicular bodies are associated with the ER and are thought to be involved in membrane recycling; they are found in a subset of dendritic spines and were present in two of the 13 somatic spines comprising the mat. Among the features that somatic spines share with dendritic spines is the frequent inclusion of ER (Fig. 4D,E). Approximately one-half of the somatic spines had extensive ER networks, revealed in the whole-spine example that is shown by rendering the spine transparent so that the mapped ER could be indicated (Fig. $4 F$ ).


Figure 5. Distribution of PSDs on and around an E15 spine mat. $A$, Only four of the 13 spines had a PSD ( yellow), although a fifth PSD was located on the soma membrane nearby. $B-D$, All PSDs had the traditional structure, including a postsynaptic thickening (arrowheads) and closely apposed presynaptic membrane. None of the PSDs on somatic spines was associated with a distinct spine head. Scale bars: $A, 500 \mathrm{~nm} ; D, 200 \mathrm{~nm}$. 

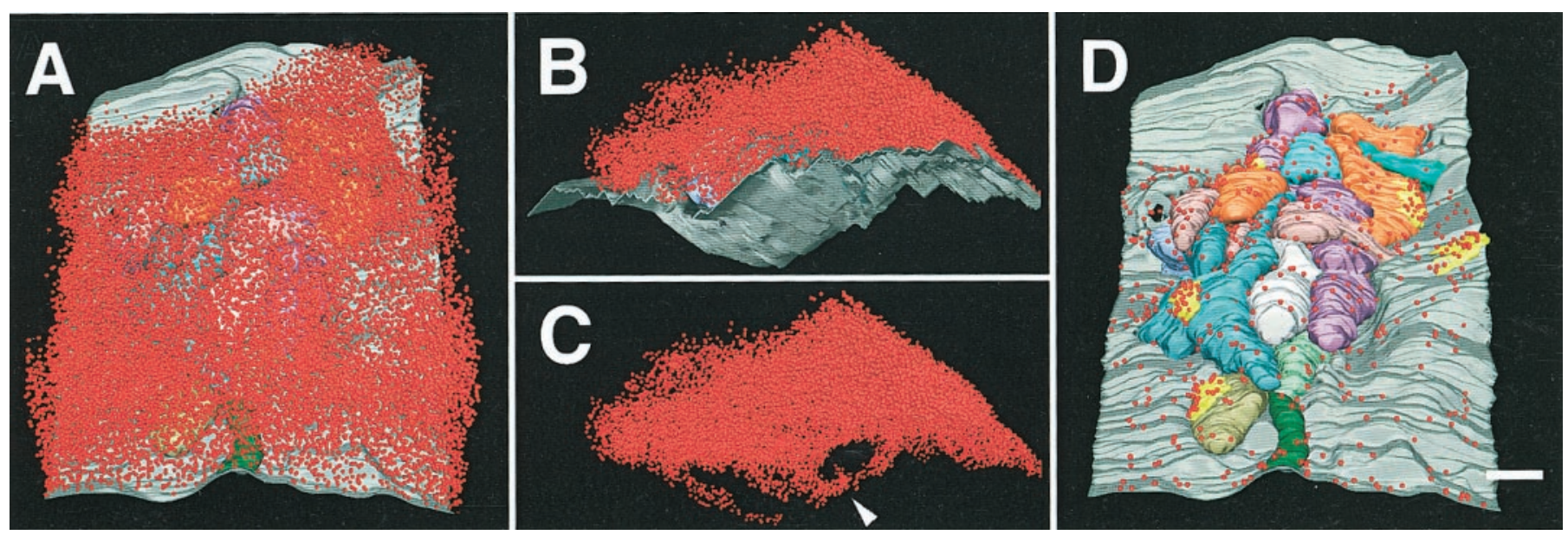

Figure 6. Distribution of synaptic vesicles in the presynaptic calyx overlaying the E15 spine mat. $A$, All synaptic vesicles in the serial sections containing the spine mat were marked individually $(r e d)$ and added to the reconstructed spine mat image from Figure $2 A$. $B$, Side view indicating the vertical distribution of the vesicle population. $C$, Side view with the postsynaptic membrane removed, illustrating vesicle-containing structures (arrowhead) extending into the spine mat. $D$, Deleting all synaptic vesicles $\geq 5 \mathrm{~nm}$ from the presynaptic membrane to reveal potentially docked vesicles at release sites left significant concentrations over the PSDs (yellow), as expected. In addition, scattered vesicles remained over much of the spine surface area, suggesting possible docked vesicles even in the absence of juxtaposed PSDs. Scale bar, $500 \mathrm{~nm}$. The artificial diagonal border of vesicles in the top right corners of $B$ and $C$ is attributable to the boundary of the visualized volume, not the perimeter of the calyx.

\section{PSDs on spines and the proximity of synaptic vesicles}

A characteristic feature of dendritic spines is the presence of at least one PSD (Harris, 1999). Transmitter release from the presynaptic bouton is thought to occur precisely over the PSD. Somatic spines on E15 ciliary neurons, however, are enveloped by the presynaptic calyx, with synaptic vesicles lining the calyx membrane. This arrangement, together with occasional "omega-shaped" profiles being discovered in the adjacent presynaptic membrane, suggested that transmitter release may occur onto somatic spines even in the absence of a PSD (Shoop et al., 1999).

Only four of the 13 somatic spines comprising the E15 mat that was examined here had a PSD (Fig. 5A). An additional PSD was found on the soma membrane immediately adjacent to the spine mat. The fine structure of the PSDs on somatic spines appeared identical to those on dendritic spines both with respect to the dimensions of the postsynaptic thickening and the presence of immediately juxtaposed (possibly "docked") synaptic vesicles on the presynaptic side (Fig. $5 B-D$ ). The position of the PSD on a somatic spine, however, was highly variable, in contrast to PSDs on dendritic spines that typically are found on a spine head.

Synaptic vesicles were mapped in the overlying presynaptic calyx and were added to the reconstructed spine mat image (Fig. $6 A$ ). The huge number of vesicles almost obscures the underlying spines. The side views with (Fig. $6 B$ ) and without (Fig. $6 C$ ) the postsynaptic membrane present reveal the deep penetration of vesicle-containing structures into the spine mat, in some cases being interdigitated with the spines themselves. Vesicles docked at release sites should be in close association with the presynaptic membrane. Deleting all vesicles from the image that were $\geq 5 \mathrm{~nm}$ from the presynaptic membrane showed a residual vesicle population with a nonuniform distribution (Fig. 6D). Local concentrations of vesicles were associated with PSDs, as expected for traditional release sites (Harris, 1999); vesicles were distributed also, however, over spines lacking PSDs, although more sparsely. Approximately one-fourth of the total spine surface present in the E15 mat that was examined was juxtaposed directly to presynaptic membrane lined with synaptic vesicles (Table 1).
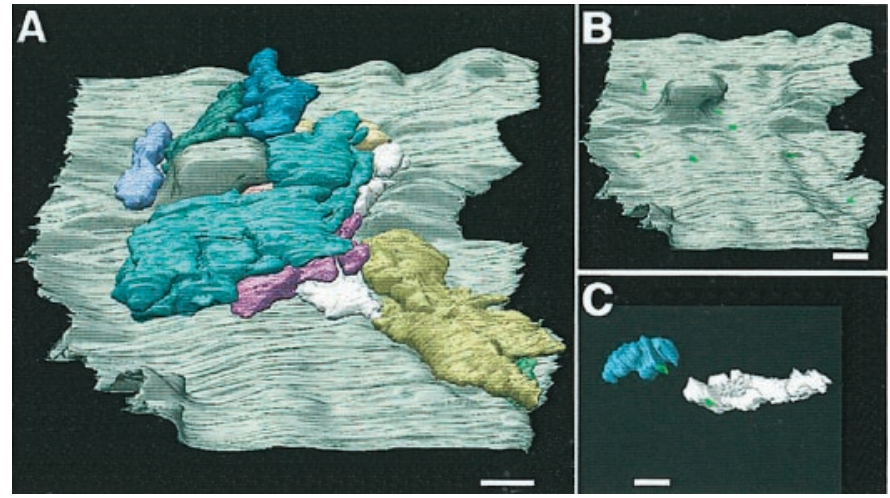

Figure 7. A nearly complete spine mat reconstructed from an adult ciliary neuron. $A$, The spine mat is shown with spines individually colorcoded (in this and subsequent figures) against the soma membrane (gray) covering a $24 \mu \mathrm{m}^{2}$ area. $B$, The attachment sites of the spine necks are shown in green after the spines were removed from the reconstructed area shown in $A$. A block-like outpocketing of the soma membrane, clearly different from the spines, is also present; only one such outpocketing was found among the several tomograms that were examined. $C$, Individually reconstructed spines have morphologies as diverse as those at E15. Scale bars: $A, B, 500 \mathrm{~nm} ; C, 200 \mathrm{~nm}$.

\section{An adult spine mat}

An adult spine mat was examined to determine whether it differed significantly from the E15 mat described above. Serial thick sections provided a three-dimensional tomographic reconstruction of an almost complete spine mat on a ciliary neuron from a 2-year-old chicken. Ten spines were resolved completely within the adult mat (Fig. 7A). Three additional separate spine segments were found, suggesting one to three more spines may have been present on the fringe of the mat and extended segments into the region that was analyzed. Each of the complete spines was connected to the soma by a narrow spine neck, and the points of attachment were distributed throughout the indented area underlying the mat (Fig. $7 B$ ) as found for the E15 mat. (A block-like outpocketing was apparent in this one tomograph; it was clearly different from spines in having no constriction where it emerged 



Figure 8. Distribution of synaptic vesicles in the presynaptic calyx overlaying an adult spine mat. $A$, All synaptic vesicles in the serial sections encompassing the spine mat were marked individually (red) and are shown superimposed on the spine mat from Figure 7. None of the spines has PSDs; unlike the situation at the embryonic spine mat, very few vesicles overlay the individual adult spines. $B$, The same view as in $A$ showing the synaptic vesicles after the spines have been removed. The large pool of synaptic vesicles in the lower right quadrant lies adjacent to the spine mat. Scale bars, $500 \mathrm{~nm}$.

from the soma.) The morphologies of the 10 complete adult spines showed considerable variation (Fig. 7C) as found for E15 spines, and the range of shapes was similar to that in the embryonic mat. The mean length $(2.6 \pm 0.7 \mu \mathrm{m}$; mean $\pm \mathrm{SEM}, n=10)$ was not significantly different from that found for embryonic spines (cf. Table 1), nor was the mean volume $\left(0.07 \pm 0.02 \mu \mathrm{m}^{3}\right)$, the mean surface area $\left(1.2 \pm 0.4 \mu \mathrm{m}^{2}\right)$, or the mean number of branches per spine $(2.4 \pm 0.4)$ significantly different. Thus in most respects the morphological features of the adult spine mat closely resembled those of the embryonic mat.

One difference, however, between the adult spine mat that was analyzed and the E15 mat described above was that adult spine necks had a smaller mean cross-sectional area and correspondingly smaller mean neck diameter $(0.12 \pm 0.01 \mu \mathrm{m})$ than did spines in the E15 mat (see above). This indicates that the constraints on chemical exchange between spine lumen and soma are likely to be preserved throughout adulthood and even may be strengthened over those seen in late stage embryos. Another difference between the embryonic and adult spine mats was that the latter was not overlaid extensively by presynaptic structures packed with synaptic vesicles. Mapping individual synaptic vesicles in the sections showed that they were concentrated in pools at the periphery of the mat (Fig. 8). A much sparser distribution of vesicles was found over most of the spines themselves. A third difference between the E15 and adult mats was that no PSDs were found on the adult spines. This is not likely to be a detection problem, because the ultrathin electronic slices made possible by the computer-reconstructed back projections allow PSDs to be detected readily, if properly stained. Most randomly chosen thin sections from adult ganglia analyzed by traditional EM showed examples of spine mats seemingly devoid of PSDs and adjacent synaptic vesicles (Fig. 9A,B), as seen for the reconstructed mat. Examples could be found, however, of thin section EM showing occasional PSDs on adult spines and nearby concentrations of synaptic vesicles (Fig. 9C,D). Some adult spines also contained ER, as did embryonic spines. The results indicate variation among adult spine mats but demonstrate that adult spines retain many of the features of spines in late embryogenesis. Notably, adult spines retain a bottle neck connection to the soma and often lack a PSD, as are true of embryonic spines.

\section{DISCUSSION}

The present account offers the first complete anatomical description of a somatic spine mat. The grouping of spines in a tightly interwoven mat folded down against the soma is clearly different from the normal arrangement of dendritic spines spaced at dis- crete intervals along the dendritic shaft (Harris and Kater, 1994). Although dendritic spines can act as independent units both with respect to synaptic activation and long-term synaptic changes, the somatic spines described here almost certainly are intended to function as a group. Synchronous activation would be expected from the single continuous presynaptic calyx enveloping the postsynaptic neuron and from the release of vesicles into a confined space occupied by multiple spines. The bottleneck of somatic spines, however, seems to be intended to constrain the chemical consequences of synaptic signaling to the sites of origin, e.g., calcium influx, as is the case for dendritic spines.

Only two spines mats were examined here in near entirety, but the results were remarkably consistent with respect to spine number, shape, configuration, and soma attachment despite the vastly different ages: embryonic versus late adult. Tomographic analysis of several thick sections from one additional adult spine mat and of individual thick sections from each of four additional embryonic spine mats (data not shown) yielded results completely consistent with those reported here, and the tomographic reconstructed cross sections were similar in complexity and content to $>200$ randomly selected thin sections through embryonic spine mats and $>50$ through adult spine mats analyzed by traditional EM. The reconstructed sizes of the two mats that were analyzed in detail were typical of those revealed by fluorescence labeling of spine constituents on the neurons (Shoop et al., 1999, 2000). These considerations provide some assurance that the morphological features that have been described are likely to be representative of spine mats on ciliary neurons.

In most respects the embryonic somatic spines that were analyzed here were morphologically similar to dendritic spines. The average volume of the spines $\left(0.11 \pm 0.06 \mu \mathrm{m}^{3}\right)$ was well within the range found for dendritic spines $\left(0.06-0.8 \mu \mathrm{m}^{3}\right.$; Harris and Stevens, 1988, 1989; Chicurel and Harris, 1992). The mean length of the somatic spines $(3.0 \pm 2.2 \mu \mathrm{m})$ and surface area $(1.6 \pm 1.3$ $\left.\mu \mathrm{m}^{2}\right)$ also lay within ranges reported for dendritic spines $(0.2-6.5$ $\mu \mathrm{m}$ in length; 0.1-5.0 $\mu \mathrm{m}^{2}$ area; Spacek and Hartmann, 1983; Harris and Kater, 1994). The average diameter and crosssectional area of the E15 spine neck were $\sim 0.2 \mu \mathrm{m}$ and $0.03 \mu \mathrm{m}^{2}$, respectively, for somatic spines. Notably, the fractional variations $( \pm$ SEM) in cross-sectional area and mean diameter for the spine neck were much smaller than the fractional variation in any of the other morphological parameters that had been measured. This implies that neck formation is a tightly regulated process and that it may use mechanisms also operative at dendritic spines that have neck diameters similar in size to those seen here (Harris and 

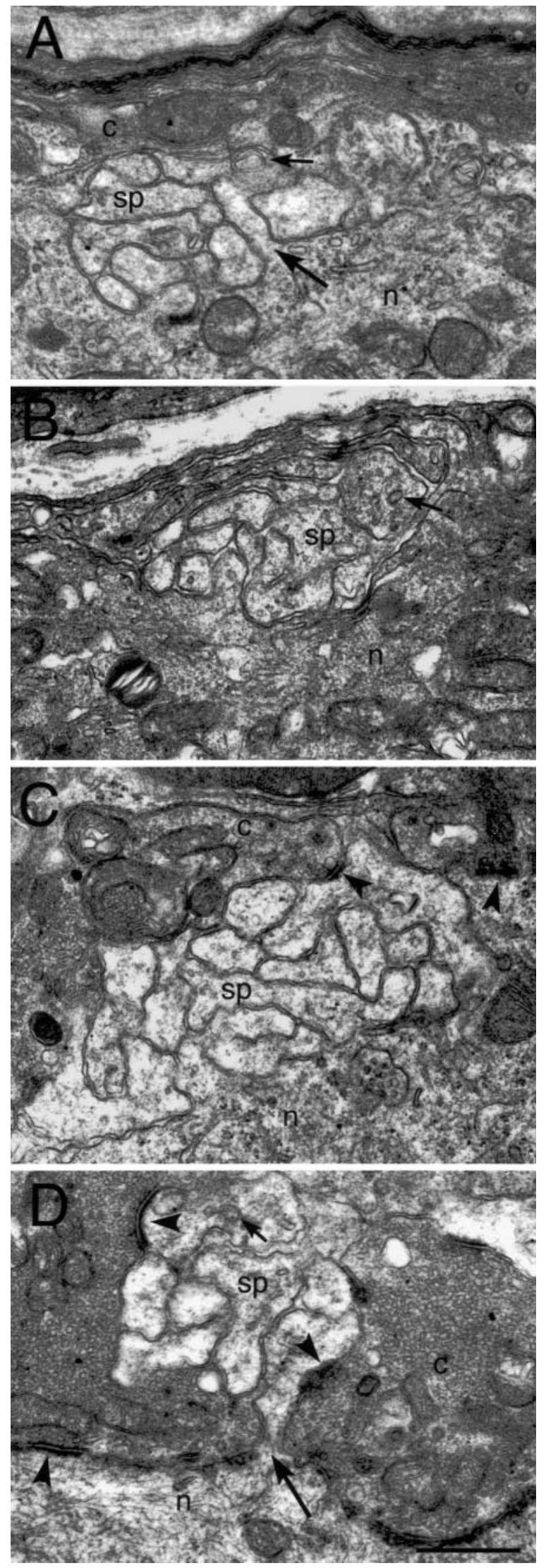

Figure 9. EM thin sections of adult ciliary neurons showing spines mats. $A$, $B$, Spine mats lying between a neuron $(n)$ and the presynaptic calyx $(c)$. Although the spines $(s p)$ have ER (small arrows), in these examples they do not have PSDs and are not adjacent to presynaptic structures with
Kater, 1994). The adult somatic spines that were analyzed displayed morphological properties within the ranges found for embryonic spines but had slightly smaller neck diameters. If this is a general feature of adult spines, it would imply an additional restriction in spine access to the soma. Other similarities between somatic spines (both embryonic and adult) and dendritic spines were the presence of ER and occasional multivesicular bodies, suggesting machinery for calcium regulation and possibly membrane turnover (Spacek and Harris, 1997).

One difference between the somatic spines that have been described here and most dendritic spines is that the former usually had multiple branches. Branching is rare for dendritic spines in most brain regions that have been examined but can be found occasionally. Approximately $90 \%$ of proximal dendritic spines are branched in the CA3 region of the hippocampus (Chicurel and Harris, 1992). The biggest difference between somatic and dendritic spines, however, is that only a minority of somatic spines had a PSD. All dendritic spines are thought to have at least one PSD, which usually is considered a defining feature of a spine. The conclusion that most somatic spines on ciliary neurons lack PSDs was made possible by imaging the entire surface area of multiple individual spines and by examining embryonic as well as adult spines. The quality of the images, particularly in the case of the E15 spine mat, was such that PSDs when present could be recognized readily.

Previous studies have demonstrated that the abundant $\alpha 7$ nAChRs found on ciliary neurons are concentrated on somatic spines and are absent from PSDs (Jacob and Berg, 1983; Loring et al., 1985; Shoop et al., 1999), although the receptors contribute significantly to synaptic currents (Zhang et al., 1996; Ullian et al., 1997; Chang and Berg, 1999). A significant proportion of the $\alpha 3^{*}$-nAChRs on ciliary neurons also may be confined to the somatic spines at non-PSD sites (Ullian et al., 1997; Shoop et al., 1999), although certainly some are concentrated in PSDs as well (Jacob et al., 1984; Williams et al., 1998). How such receptors are accessed by transmitter raises questions about the sites of transmitter release. Two possibilities have been proposed: lateral diffusion from traditional release sites over PSDs and release from nontraditional sites along the calyx directly onto the spines at non-PSD sites (Shoop et al., 1999). Although lateral diffusion might seem slow, the distances involved are compatible with the rapid rise time of the synaptic currents (Zhang et al., 1996; Ullian et al., 1997). This is because the somatic spines comprising a single mat are packed into an area of $\sim 20 \mu \mathrm{m}^{2}$ and because several PSDs are distributed throughout the mat.

The alternative possibility is supported by the close association of synaptic vesicles overlaying the spines and by the observation of occasional membrane profiles (omega shapes) suggestive of vesicles trapped in the process of exocytosis over the spines with no PSDs nearby (Shoop et al., 1999). Although the present study does not resolve this issue for embryonic spines, it does suggest that lateral diff usion must suffice in the adult if $\alpha 7-\mathrm{nAChRs}$ are to be activated on most of the spines. This follows from the observation that almost an entire adult spine mat was found to be

appreciable numbers of vesicles. A spine neck is indicated by the large arrow in $A$. C, D, Spine mats showing both ER (small arrow) and synaptic vesicles in the overlying calyx (c). Multiple PSDs are indicated (arrowheads). The spine mat in $D$ is surrounded totally by a large pool of vesicles, in sharp contrast to the almost complete lack of vesicles in most thin sections through the spine mats in $A$ and $B$. Scale bar, $500 \mathrm{~nm}$. 
devoid of PSDs and, moreover, that presynaptic concentrations of synaptic vesicles were confined primarily to the periphery of the spine mat in that case. The grouping of somatic spines into tightly packed mats may increase the efficiency of transmission; release of a few vesicles from distributed sites should be able to access receptors on most of the spines within the small volume containing the spine mat. Efficiency of this sort may be essential for maintaining the reliability and high-frequency firing capability of the synapse (Dryer, 1994).

It is also possible that much of the signaling responsibility of $\alpha 7$-nAChRs, particularly in the adult, does not require rapid activation but rather takes advantage of the fact that the receptors respond to choline as an agonist (Mandelzys et al., 1995; Alkondon et al., 1997). Acetylcholinesterase is distributed throughout the calyx synapse and around membrane specializations now recognized to be spine mats (Olivieri-Sangiacomo et al., 1983a,b). Hydrolysis of released ACh to produce free choline could activate the $\alpha 7$-nAChRs over a longer time course easily compatible with diffusion times. Although the receptors normally display rapid desensitization when challenged with high concentrations of ACh or nicotine (Zorumski et al., 1992; Alkondon and Albuquerque, 1993), lower agonist levels can produce more sustained responses (Zhang et al., 1994).

The maintenance of somatic spine mats throughout adulthood, even when synaptic vesicles are relegated to peripheral positions, suggests that the receptor-laden spine structure is essential for some aspect of synaptic signaling other than simple electrical transmission. Most likely it involves receptor-mediated calcium influx that may need compartmentalization to achieve adequate local concentrations for regulatory purposes and yet avoid cytotoxicity. The fact that the calyx synapse also has an electrical component supplementing nicotinic transmission (Martin and Pilar, 1964) may impose special requirements for control of postsynaptic calcium resulting from ganglionic transmission. Imaging studies have demonstrated that low-frequency synaptic stimulation enables $\alpha 7$-nAChRs to produce local calcium transients confined to the spines (Shoop et al., 2001). Receptormediated calcium influx over the short term can regulate $\alpha 7$ nAChR responses (Liu and Berg, 1999). Over the long term, repeated synaptic stimulation of nicotinic receptors exerts calcium-dependent transcriptional regulation in the neurons, changing the levels of certain transcripts (Chang and Berg, 2001). How somatic spines form and become stabilized and how nicotinic receptors become localized on the spines remain issues for future investigation.

\section{REFERENCES}

Alkondon M, Albuquerque EX (1993) Diversity of nicotinic acetylcholine receptors in rat hippocampal neurons. I. Pharmacological and functional evidence for distinct structural subtypes. J Pharmacol Exp Ther 265:1455-1473.

Alkondon M, Pereira EFR, Cortes WS, Maelicke A, Albuquerque EX (1997) Choline is a selective agonist of $\alpha 7$ nicotinic acetylcholine receptors in rat brain neurons. Eur J Neurosci 9:2734-2742.

Bundman MC, Pico RM, Gall CM (1994) Ultrastructural plasticity of the dentate gyrus granule cells following recurrent limbic seizures. I. Increase in somatic spines. Hippocampus 4:601-610.

Chang K, Berg DK (1999) Dependence of circuit function on nicotinic acetylcholine receptors containing $\alpha 7$ subunits. J Neurosci 19: 3701-3710.

Chang K, Berg DK (2001) Voltage-gated channels block nicotinic regulation of CREB phosphorylation and gene expression in neurons. Neuron 32:855-865.

Chiappinelli VA, Giacobini E (1978) Time course of appearance of $\alpha$-bungarotoxin binding sites during development of chick ciliary ganglion and iris. Neurochem Res 3:465-478.

Chicurel ME, Harris KM (1992) Three-dimensional analysis of the structure and composition of CA3 branched dendritic spines and their synaptic relationships with mossy fiber boutons in the rat hippocampus. J Comp Neurol 325:169-182.

Conroy WG, Berg DK (1995) Neurons can maintain multiple classes of nicotinic acetylcholine receptors distinguished by different subunit compositions. J Biol Chem 270:4424-4431.

De Stefano ME, Luzzatto AC, Mugnaini E (1993) Neuronal ultrastructure and somatostatin immunolocalization in the ciliary ganglion of chicken and quail. J Neurocytol 22:868-892.

Dryer S (1994) Functional development of the parasympathetic neurons of the avian ciliary ganglion: a classic model system for the study of neuronal differentiation and development. Prog Neurobiol 43:281-322.

Harris KM (1999) Structure, development, and plasticity of dendritic spines. Curr Opin Neurobiol 9:343-348.

Harris KM, Kater SB (1994) Dendritic spines: cellular specializations imparting both stability and flexibility to synaptic function. Annu Rev Neurosci 17:341-371.

Harris KM, Stevens JK (1988) Dendritic spines of rat cerebellar Purkinje cells: serial electron microscopy with reference to their biophysical characteristics. J Neurosci 8:4455-4469.

Harris KM, Stevens JK (1989) Dendritic spines of CA1 pyramidal cells in the rat hippocampus: serial electron microscopy with reference to their biophysical characteristics. J Neurosci 9:2982-2997.

Hessler D, Young SJ, Carragher BO, Martone M, Lamont S, Whittaker M, Milligan RA, Masliah E, Hinshaw JE, Ellisman MH (1992) Programs for visualization in three-dimensional microscopy. NeuroImage 1:55-67.

Jacob MH, Berg DK (1983) The ultrastructural localization of $\alpha$-bungarotoxin binding sites in relation to synapses on chick ciliary ganglion neurons. J Neurosci 3:260-271.

Jacob MH, Berg DK, Lindstrom JM (1984) Shared antigenic determinant between the Electrophorus acetylcholine receptor and a synaptic component on chicken ciliary ganglion neurons. Proc Natl Acad Sci USA 81:3223-3227.

Liu Q-S, Berg DK (1999) Actin filaments and the opposing actions of $\mathrm{CaM}$ kinase II and calcineurin in regulating $\alpha 7$-containing nicotinic receptors on chick ciliary ganglion neurons. J Neurosci 19:10280-10288.

Loring RH, Zigmond RE (1987) Ultrastructural distribution of ${ }^{125} \mathrm{I}-$ toxin $F$ binding sites on chick ciliary neurons: synaptic localization of a toxin that blocks ganglionic nicotinic receptors. J Neurosci 7:2153-2162.

Loring RH, Dahm LM, Zigmond RE (1985) Localization of $\alpha$-bungarotoxin binding sites in the ciliary ganglion of the embryonic chick: an autoradiographic study at the light and electron microscopic level. Neuroscience 14:645-660.

Mandelzys A, De Koninck P, Cooper E (1995) Agonist and toxin sensitivities of ACh-evoked currents in neurons expressing multiple nicotinic ACh receptor subunits. J Neurophysiol 74:1212-1221.

Martin AR, Pilar G (1964) An analysis of electrical coupling at synapses in the avian ciliary ganglion. J Physiol (Lond) 171:454-475.

Matus A (2000) Actin-based plasticity in dendritic spines. Science 290:754-758

Olivieri-Sangiacomo C, Del Fa A, Gangitano C (1983a) Acetylcholinesterase localization at synapses in chick embryo ciliary ganglion. Experientia 39:598-600.

Olivieri-Sangiacomo C, Del Fa A, Gangitano (1983b) Developmental distributive pattern of acetylcholinesterase in chick embryo ciliary ganglion. Brain Res 283:61-69.

Perkins GA, Renken CW, Song JY, Frey TG, Young SJ, Lamont S, Martone ME, Lindsey S, Ellisman MH (1997) Electron tomography of large, multicomponent biological structures. J Struct Biol 120:219-227.

Piezzi RS, Rodriguez-Echandia EL (1968) Studies on the pararenal ganglion of the toad Bufo arenarum Hensel. I. Its normal fine structure and histochemical characteristics. Z Zellforsch Mikrosk Anat 88:180-186.

Ramon y Cajal S (1893) Neue darstellung vom histologischen bau des centralnervesystem. Arch Anat Entwick 1:319-418.

Robertson GN, Jackson PC (1996) Axosomatic synapses of the rat ciliary ganglion. Synapse 22:269-280.

Sabatini BL, Maravall M, Svoboda K (2001) $\mathrm{Ca}^{2+}$ signaling in dendritic spines. Curr Opin Neurobiol 11:349-356.

Seress L, Ribak CE (1985) A substantial number of asymmetric axosomatic synapses is a characteristic of the granule cells of the hippocampal dentate gyrus. Neurosci Lett 56:21-26.

Shoop RD, Martone ME, Yamada N, Ellisman MH, Berg DK (1999) Neuronal acetylcholine receptors with $\alpha 7$ subunits are concentrated on somatic spines for synaptic signaling in embryonic chick ciliary ganglia. J Neurosci 19:692-704.

Shoop RD, Yamada N, Berg DK (2000) Cytoskeletal links of neuronal acetylcholine receptors containing $\alpha 7$ subunits. J Neurosci 20:4021-4029.

Shoop RD, Chang KT, Ellisman MH, Berg DK (2001) Synaptically driven calcium transients via nicotinic receptors on somatic spines. J Neurosci 21:771-781. 
Smolen A (1988) Morphology of synapses in the autonomic nervous system. J Electron Microsc Tech 10:187-204.

Soto GE, Young SJ, Martone ME, Deerinck TJ, Lamont S, Carragher BO, Hama K, Ellisman MH (1994) Serial section electron tomography: a method for three-dimensional reconstruction of large structures. NeuroImage 1:230-243.

Spacek J, Harris KM (1997) Three-dimensional organization of smooth endoplasmic reticulum in hippocampal CA1 dendrites and dendritic spines of the immature and mature rat. J Neurosci 17:190-203.

Spacek J, Hartmann M (1983) Three-dimensional analysis of dendritic spines. I. Quantitative observations related to dendritic spine and synaptic morphology in cerebral and cerebellar cortices. Anat Embryol (Berl) 167:289-310.

Stirling RV, Bliss TVP (1978) Observations on the commissural projection to the dentate gyrus in the reeler mutant mouse. Brain Res 150:447-465

Ullian EM, McIntosh JM, Sargent PB (1997) Rapid synaptic transmission in the avian ciliary ganglion is mediated by two distinct classes of nicotinic receptors. J Neurosci 17:7210-7219.

Vernallis AB, Conroy WG, Berg DK (1993) Neurons assemble acetylcholine receptors with as many as three kinds of subunits while maintaining subunit segregation among receptor subtypes. Neuron 10: 451-464.
Watanabe H (1971) Adrenergic nerve elements in the hypogastric ganglion of the guinea pig. Am J Anat 130:305-330.

Wenzel J, Otani S, Desmond NL, Levy WB (1994) Rapid development of somatic spines in stratum granulosum of the adult hippocampus in vitro. Brain Res 656:127-134.

Williams BM, Tamburni MK, Schwartz Levey M, Bertrand S, Bertrand D, Jacob MH (1998) The long internal loop of the $\alpha 3$ subunit targets $\mathrm{nAChRs}$ to subdomains within individual synapses on neurons in vivo. Nat Neurosci 1:557-562.

Wilson Horch HL, Sargent PB (1995) Perisynaptic surface distribution of multiple classes of nicotinic acetylcholine receptors on neurons in the chicken ciliary ganglion. J Neurosci 15:7778-7795.

Yuste R, Majewska A, Holthoff K (2000) From form to function: calcium compartmentalization in dendritic spines. Nat Neurosc 3:653-659.

Zhang Z-W, Vijayaraghavan S, Berg DK (1994) Neuronal acetylcholine receptors that bind $\alpha$-bungarotoxin with high affinity function as ligandgated ion channels. Neuron 12:167-177.

Zhang Z-W, Coggan JS, Berg DK (1996) Synaptic currents generated by neuronal acetylcholine receptors sensitive to $\alpha$-bungarotoxin. Neuron 17:1231-1240.

Zorumski CF, Thio LL, Isenberg KE, Clifford DB (1992) Nicotinic acetylcholine currents in cultured postnatal rat hippocampal neurons. Mol Pharmacol 41:931-936. 